\title{
Experimental and Computational Techniques for the Analysis of Proton Beam Propagation THROUGH A TARGET STACK \\ L.A. Marus ${ }^{1}$, J.W. Engle ${ }^{2}$, K.D. John, E.R. Birnbaum, and F.M. Nortier
}

Los Alamos National Laboratory

P.O. Box 1663, Los Alamos, New Mexico, 87545

${ }^{1,2}$ Corresponding authors, Email: lamarus@lanl.gov, jwengle@lanl.gov

\begin{abstract}
Proton beam energy, energy straggling, and intensity in thick stacks of target materials at the Los Alamos Isotope Production Facility were investigated using the foil activation technique and computational simulations. Isotopic yield measurements of irradiated foils from several recent experiments used to determine these quantities were compared with the predictions of MCNP6 and TRIM codes, and with Andersen \& Ziegler's semi-empirical formalism. Differences between code predictions and experimental data were examined. Methods for computational simulation of energy propagation agree well with one another and were able to accurately predict the proton beam's energy for a limited range. Predictions were accurate when degrading from an initial energy of $100 \mathrm{MeV}$ down to approximately $50 \mathrm{MeV}$, but struggled to represent measured data well at lower energies.
\end{abstract}

\section{Introduction}

Particle transport codes which employ measured or modeled nuclear data to determine the outcome of individual ion-atom probabilistic events are widely used for predicting radiation interaction with materials. One of the more versatile of these codes, Monte Carlo N-Particle (MCNP6) transport code, is based on cross sections for particle interactions [1]. The SRIM/TRIM program suite [2] is based on the parameterization of quantum physical models to experimental data including stopping powers, and is in this way not dissimilar to the semi-empirical formalisms more simply employed by Anderson and Ziegler [3]. As a predictive tool, such calculations are instrumental in the design of radionuclide production targets. These tools are used to calculate energy propagation which aids in cross section measurement experiments. Validation of these calculations against experimental data is therefore critical. We routinely apply MCNP6, TRIM, and A\&Z in experiments using the $100 \mathrm{MeV}$ proton beam at the Los Alamos Isotope Production Facility (IPF).

Characterization of the proton energy and intensity as a function of depth in target material is not straight-forward at IPF, where the proton beam is completely stopped by tens of grams per $\mathrm{cm}^{2}$ target stacks. A stacked-foil activation technique [4] is a convenient method for monitoring the beam energy and intensity at specific locations within a target where Faraday cups and other direct energy measurements may not be possible. In this approach, thin, pure metal foils with well-known production cross sections, acting as targets for nuclear reactions, are irradiated in an experimental stack. By correlating predicted isotopic yields calculated using particle transport codes with yields that are experimentally measured, the effective energy and proton fluence can be determined. Accurate energy and current determinations within a specific geometry afford greater certainty for cross section measurements. These cross section measurements can, in turn, be used to optimize target designs 
for isotope production by exploiting the most productive energy regions of nuclear excitation functions.

Most $(p, x)$ nuclear formation cross sections peak below $100 \mathrm{MeV}$ and their corresponding isotope yields are sensitive to protons with energy in the range of 0-100 MeV. Small variations or straggling in the beam energy incident on a target stack can have drastic effects on the quantity and purity of accelerator produced isotopes. In this work we compared predictions of ${ }^{22} \mathrm{Na}$ production in aluminum monitor foils in a stacked foil experiment with experimentally measured isotope yields. This comparison provided insight into the validity of these computational tools and suggests careful planning is necessary when designing target stacks which attempt to use the entire effective energy range of the IPF proton beam for radioisotope production in thick targets. These considerations are also applicable to other high-energy production facilities including BLIP (US), INR (Russia), ARRONAX (France), TRIUMF (Canada), and iThemba (South Africa).

In recent years, several proton irradiation experiments [5]-[9] were conducted at IPF utilizing the stacked foil technique, some with the purpose of measuring cross sections in thin terbium foils $[7,10]$. Within each of these stacks, aluminum monitor foils were interspersed with the intent of measuring the proton beam intensity at various depths within the target stack. These foils were examined in this work.

In the experiments, the foil stacks were irradiated with a nominal, primary beam energy of $100 \mathrm{MeV}$, derived from accelerator tuning parameters. Initial predictions of ${ }^{22} \mathrm{Na}$ yields using these beam conditions showed significant discrepancy for downstream aluminum foils at the rear of the target stack. This strongly suggested that the actual beam energy was lower than $100 \mathrm{MeV}$.

This work established motivation for the implementation of a direct, time-of-flight measurement of the proton beam energy upstream of the target stack. While a time-of-flight measurement improved the agreement for the predicted ${ }^{22} \mathrm{Na}$ production and experimentally measured isotopic yields in some of the foils, it did not fully resolve the observed discrepancy for the entire foil target stack. This paper describes an effort to reconcile measured and computational data.

\section{Materials and Methods}

The ${ }^{27} \mathrm{Al}(p, x)^{22} \mathrm{Na}$ reaction has a well-characterized energy-dependent production cross section, see FIGURE 1, in the energy range of interest, 30-100 MeV. Due to the extent of its characterization compared with other reactions, this reaction is almost exclusively used for proton intensity monitoring purposes at these energies [4, 5]. We used IAEA NDS recommended cross sections for the ${ }^{27} \mathrm{Al}(\mathrm{p}, \mathrm{x})^{22} \mathrm{Na}$ reaction, sourced from the IAEA charged particle database [11]. Since no uncertainties are presently assigned to IAEA recommended cross sections, we adopted uncertainties based on the data reported by Steyn, et al. [10] as a conservative measure. 


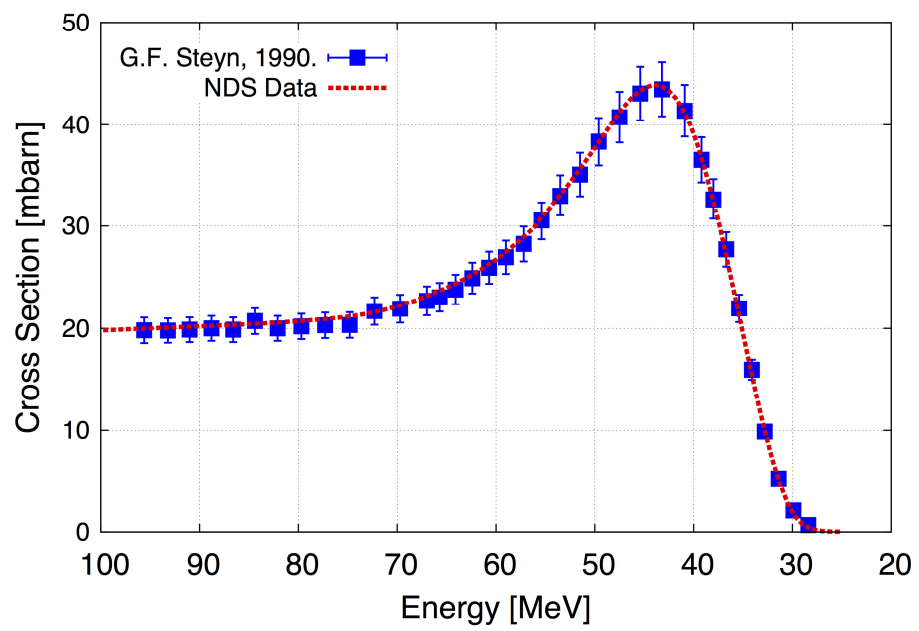

FIGURE 1: Published cross section for ${ }^{27} \mathrm{Al}(\mathrm{p}, \mathrm{x})^{22} \mathrm{Na}$ used in Al monitor foils, from NDS data [11] and from Steyn, et al. [10].

Sodium-22 has a well-defined gamma peak at $1274.5 \mathrm{keV}$ and intensity $99.94 \%$. This gamma was used to quantify the activity of ${ }^{22} \mathrm{Na}$ production experimentally. Predicted yields of ${ }^{22} \mathrm{Na}$ generated in the Al monitor foils were calculated using the aforementioned codes: MCNP6, TRIM, and A\&Z, and utilizing the IAEA recommended cross sections. These predictions were compared with experimental measurements of ${ }^{22} \mathrm{Na}$ yields.

Time-of-flight measurements revealed a primary beam energy of $99.1 \pm 0.5 \mathrm{MeV}$. The 0.5 $\mathrm{MeV}$ uncertainty is derived from an observed variation during the duration of the measurement, and is not a reflection of the error in the experimental measurement itself. The timeof-flight measurement was performed by tapping into existing beam position monitoring equipment for a frequency domain or phase measurement of the beam's micropulses. The measured value of $99.1 \mathrm{MeV}$ was used in all simulations presented herein.

\subsection{Experimental Approach}

For the purpose of monitoring the proton fluence in the measurement of $T b+p$ excitation functions, thin aluminum monitor foils $(0.25 \mathrm{~mm}$ thickness, $2.5 \times 2.5 \mathrm{~mm})$ of high purity (Goodfellow Metals, mass $0.417 \pm 0.003 \mathrm{~g}$ ) were encapsulated in $25 \mu \mathrm{m}$ thick Kapton ${ }^{\circledR}$ tape and stacked with the terbium target foils. To facilitate cross section measurements on terbium at predetermined energies, target foils and their accompanying monitor foils were separated by additional aluminum degraders, included in the design of a specially fabricated aluminum foil holder. The Kapton ${ }^{\circledR}$ enclosed Tb target foils were stacked with the Al foils and irradiated with protons at $100 \mathrm{nA}$, see FIGURE 2. 

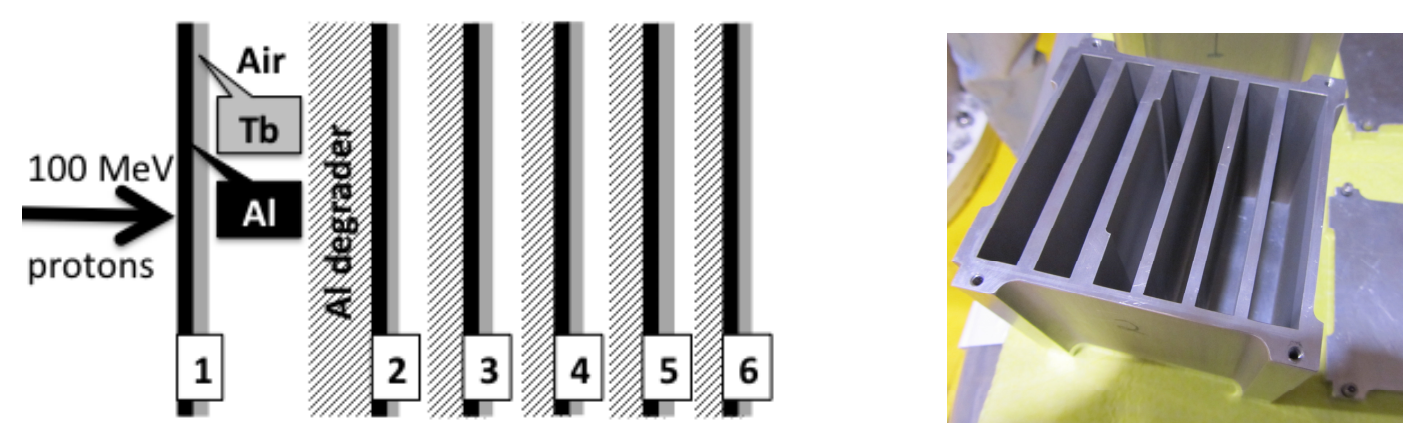

FIGURE 2: Schematic of the stacked foil target and photo of Al foil holder used in a recent Tb foil cross section experiment. Not shown in the schematic, but accounted for in calculations were the vacuum-isolation window, cooling water, and the target holder upstream of the foil stack.

Following a one hour irradiation, each Al foil was assayed using $\gamma$-spectroscopy on calibrated HPGe detectors in the Chemistry Division countroom at LANL. Peaks were identified and corrected for background using the SPECANL analysis algorithm. Details of the experimental activity determination and associated uncertainties have been reported previously [5].

\subsection{Computational Simulations}

The algorithm of the MCNP6 code designed to track the number of protons that cross each aluminum foil is the F4 volumetric tally. In order to obtain an effective fluence at each foil position, the dimensionless MCNP6 tally representing the predicted number of protons was normalized, then multiplied by the fluence calculated from the measured activity for the first foil. EQUATION 1 was used to calculate the fluence of the first foil from the measured activity [11].

$\sigma_{i}(E)=2.678 \times 10^{-10}\left[A \lambda N_{i} /\left(I \rho x\left(1-e^{-\lambda t}\right)\right)\right]$

where:

$\sigma_{i}(E)$ is the cross section for the process, [mbarn]

$A$ is the atomic mass of the target, [amu]

$N_{i}$ is the is the number of product nuclei present at End-of-Bombardment

$I$ is the average beam current, $[\mu \mathrm{A}]$

$\rho$ is the density of the target material, $\left[\mathrm{g} / \mathrm{cm}^{3}\right]$

$x$ is the target thickness, $[\mathrm{cm}]$

$\lambda$ is the decay constant, $\left[\mathrm{s}^{-1}\right]$

$t$ is the irradiation time, [s]

As the beam passes through target material, there is also a statistically driven broadening of its effective energy, the shape of which is predicted by MCNP6 and TRIM. To account for the energy distribution, we calculated an effective or weighted cross-section. It is especially important to address energy broadening in regions where the excitation function under consideration varies rapidly with energy. In the excitation function shown in FIGURE 1, a strong 
variation in the energy range from $30-65 \mathrm{MeV}$ is observed, the energy region covered by the last 3 foils in the stack.

A correction must be applied to the cross sections to account for energy straggling incident on the foil. An effective cross section for each foil is derived by weighting the excitation function by the distribution of energies incident on the foil. The incident energy takes a Gaussian shaped distribution, $w_{i}\left(E_{i}\right)$, with the tallies for each energy bin $\left(E_{i}\right)$ determining the parameters of the fit:

$w_{i}\left(E_{i}\right)=\frac{1}{\sigma_{S D} \sqrt{2 \pi}} \exp \left[-\frac{\left(E_{i}-\mu\right)^{2}}{2 \sigma_{S D}^{2}}\right]$

where $\mu$ is the mean energy and $\sigma_{S D}$ is the standard deviation from the mean energy. The Gaussian distribution was multiplied by corresponding cross sections $\left(\sigma_{i}\right)$ for each energy bin in order to find a weighted average of the cross section, $\sigma_{\text {wav }}$ :

$\sigma_{w a v}=\frac{\sum w_{i}\left(E_{i}\right) \sigma_{i}\left(E_{i}\right)}{\sum w_{i}\left(E_{i}\right)}$

\subsection{Results}

TABLE 1 and FIGURE 3 summarize the predicted mean energy and standard deviation of the mean energy incident on each aluminum monitor foil.

Given the calculated effective cross section weighted by the energy distribution and the predicted fluence at each of the foils, the predicted number of ${ }^{22} \mathrm{Na}$ atoms created and the expected activity of each foil is obtained using EQUATION 1. Results are compared with measured activities in FIGURE 4.

Unexpected deviations in the calculated yields from experimental measurements were observed for foils towards the back of the stack, and prompted further investigation. The following discussion examines the differences between the computational methods, the extent by which simulation assumptions affect calculation, and future work that is needed to fully resolve this observed phenomenon. 


\begin{tabular}{llllr}
\hline \multicolumn{2}{l}{ Mean Energy [MeV] (standard deviation) } & & & Current [nA] \\
\hline $\begin{array}{llll}\text { Foil } \\
\text { Number }\end{array}$ & MCNP6 & TRIM & A\&Z & \\
\hline 1 & & & & 100.8 \\
2 & $89.5(0.4)$ & $89.4(0.4)$ & 89.4 & 99.6 \\
3 & $80.2(0.6)$ & $79.9(0.6)$ & 79.9 & 98.1 \\
4 & $70.8(0.8)$ & $70.3(0.8)$ & 70.2 & 96.0 \\
5 & $62.5(0.9)$ & $61.9(0.9)$ & 61.8 & 93.6 \\
6 & $53.5(1.0)$ & $52.6(1.1)$ & 52.4 & 90.0 \\
\hline
\end{tabular}

TABLE 1. Comparison of the mean and standard deviation of the proton fluence energy distribution incident on each aluminum foil predicted by MCNP6, TRIM, and A\&Z computations for the Tb foil stack experiment assuming $99.1 \mathrm{MeV}$ primary beam energy.

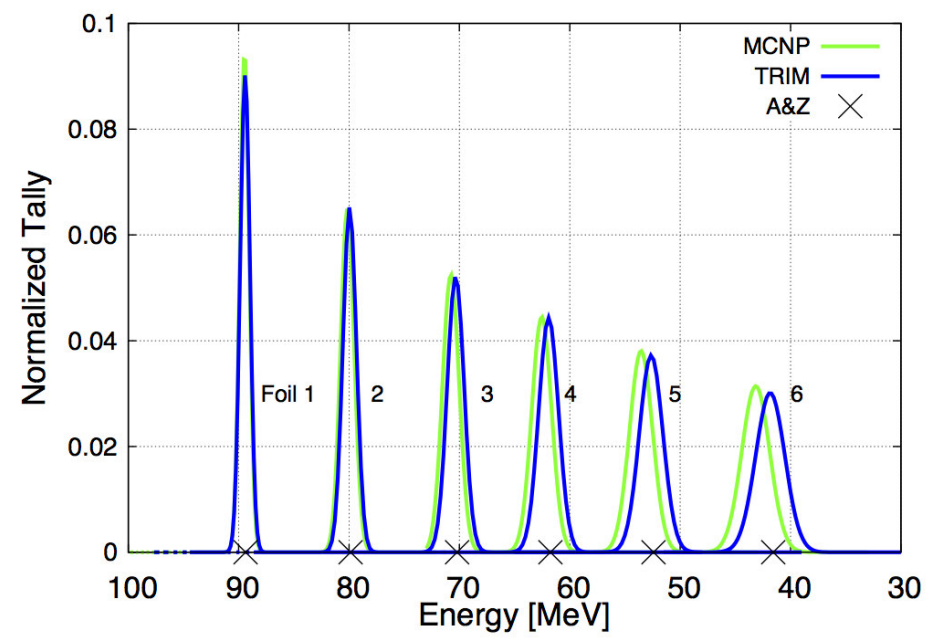

FIGURE 3: Comparison of proton fluence energy distribution in a recent Tb foil stack experiment assuming $99.1 \mathrm{MeV}$ primary beam energy using stacked foils at 6 energy positions as predicted by MCNP6, TRIM, and A\&Z computational methods. Energy distribution integral was normalized within each individual foil, not between foils. 


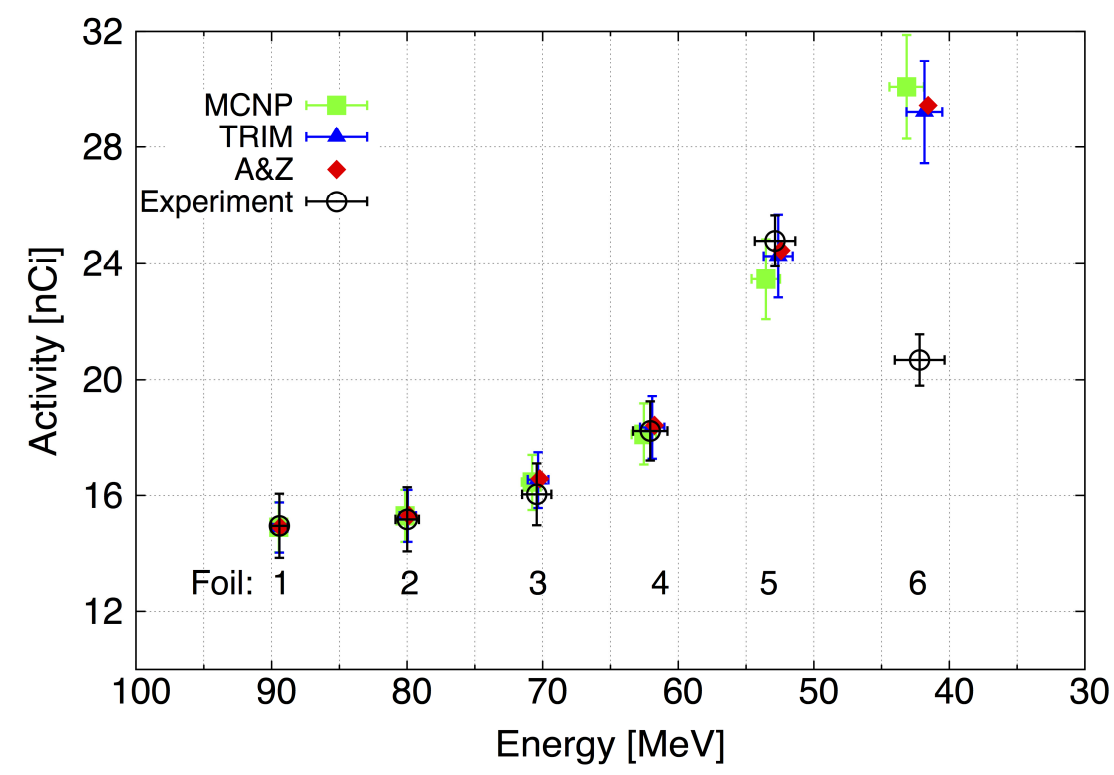

FIGURE 4: Comparison of theoretically calculated ${ }^{22} \mathrm{Na}$ activity using MCNP6, TRIM, and A\&Z versus experimentally measured ${ }^{22} \mathrm{Na}$ activity for $\mathrm{Al}$ monitor foils in Tb foil stack experiment. Plotted energy values and associated error bars indicated here are derived from an average of the computationally determined energies, adding their uncertainties in quadrature. Error bars for experimental activity measurements were derived from uncertainty associated with $\gamma$-spectroscopy counting. Error bars for computational calculations are attributed to uncertainty in cross section measurements.

\section{Discussion}

In the time frame of medium-energy experiments at the IPF, we were unable to accurately assay ${ }^{24} \mathrm{Na}$ quantities, so aluminum monitor foils afford only one long-lived isotope for evaluation, ${ }^{22} \mathrm{Na}$; a complementary method such as isotope ratio analysis [12] was not achievable for these experiments. Thus, confidence in the beam characterization by the aluminum monitor foil method was predicated on accurate predictions of the effective proton beam energy in the foil stack.

\subsection{Computational Simulation Methods}

Andersen \& Zeigler (A\&Z) is a deterministic method and also the simplest of the three computational methods considered. While the mean energy degradation can be calculated using the $A \& Z$ formalism, the beam attenuation cannot. Consequently, the $A \& Z$ method lacks the ability to account for a broadening in the beam energy that a stochastic method affords. Additionally, A\&Z does not account for nuclear recoil or contributions from secondary interactions.

TRIM uses a stochastic based method to calculate the stopping range of incident particles applying Bethe-Block formalisms. TRIM, like A\&Z, does not include contributions from nuclear recoil or contributions from secondary interactions. Computationally, TRIM is an ex- 
pensive code to run, requiring significant computational time to obtain good statistics. The code is able to calculate a broadening in the energy of the beam; however, beam attenuation predictions are much less reliable. For example, TRIM predicts the total beam attenuation in the whole stack to be less than one percent, while $7-10 \%$ is expected [13].

Note that TRIM and A\&Z methods each predicts the mean energy incident on individual foils within the foil stack, while TRIM has the additional benefit of calculating the energy straggling. Independent predictions made by TRIM and A\&Z are reported as "calculated". Since the use of both these two methods yields inaccurate beam attenuation data, MCNP6 results were used in calculations for the prediction of the ${ }^{22} \mathrm{Na}$ activity in aluminum foils instead.

MCNP6 is arguably the most sophisticated approach to modeling the physics of the experiment. For proton reactions in the relevant energy range, MCNP6 uses a stochastic procedure for calculation, adopting the Cascade-Exciton Model (CEM03.03) [14] to track particles. The physics card was enabled in the MCNP6 input to track light ion recoils. Contributions from neutron and proton secondary particle interactions were included, although their contribution was minimal. For both MCNP6 and TRIM, the proton beam was simulated as a pencil beam. In order to determine beam attenuation, MCNP6 was used to calculate the fluence of transmitted protons using optical model formalisms, taking into account potential proton removal from the beam due to outscattering and nuclear reaction events.

By updating the incident beam energy from $100 \mathrm{MeV}$ to the measured value of $99.1 \mathrm{MeV}$ an improvement in the agreement between measured and computationally derived ${ }^{22} \mathrm{Na}$ activity in the foils is achieved. This shows that obtaining a time-of-flight measurement and its associated uncertainty, although not concurrent with the experiment, permitted a more grounded investigation into other, less obvious uncertainties. The combined effort of these investigations into measured parameters improved the agreement significantly, especially in the case of the $4^{\text {th }}$ and $5^{\text {th }}$ foils. The disagreement at the $6^{\text {th }}$ foil remains unresolved.

When comparing predictions of ${ }^{22} \mathrm{Na}$ yields with experiment for the purpose of proton fluence monitoring, it is important to consider the date set as a whole. Since the calculated activity is directly proportional to the cross section, the general trend in the amount of activity produced in the foils should follow the shape of the excitation function for the ${ }^{27} \mathrm{Al}(\mathrm{p}, \mathrm{x})^{22} \mathrm{Na}$ reaction; compare the shape exemplified in FIGURE 1 with FIGURE 4. However, deviations in the calculated yields from experimental measurements were observed for foils towards the back of the stack, at energies below $50 \mathrm{MeV}$.

We conclude that the three codes utilized generally under-predict the energy loss of protons as they pass through the layers in the foil stack. Small difference between the predicted and measured incident energies at the front of the foil stack "trickle down" to produce much more pronounced shifts in the energy at foils towards the back of the foil stack. The characteristic "rolling over" in the shape of the activity curve seen in the experimental foils indicates that the $6^{\text {th }}$ foil, as seen in FIGURE 4, must have been located in the energy region below $40 \mathrm{MeV}$, i.e. below where the peak of the excitation function occurs.

The origin of discrepancy in activities between prediction and experiment at foil 6 is not yet fully understood. Potential contributors to this discrepancy include additional uncertainties 
in modeling results and experimental measurements. Note that uncertainties in experiment and computations are tied, in a complex interconnected way, since certain experimental data are used as input for calculations. Uncertainty in measured parameters like the thickness and density of the foils in the stack impacts confidence in both approaches.

There remains also an uncertainty in the actual value of the primary beam energy of the linac because concurrent time-of-flight measurement capabilities were not available at the time the foil stack experiments were conducted. Although we do not have a concurrent measurement, recent historical beam energy data indicate that it is reasonable to assume the energy at the time of the stacked foil experiments was lower than the assumed energy of $100 \mathrm{MeV}$. Computational simulations developed in this work firmly supported this assumption.

As a diagnostic, the primary beam energy was changed from $99.1 \pm 0.5 \mathrm{MeV}$ to $98.6 \mathrm{MeV}$, which is the lowest value in the measured historical beam energy data. While this exercise showed some improvement in the agreement between predicted and measured activities of

${ }^{22} \mathrm{Na}$ yields for the $5^{\text {th }}$ and $6^{\text {th }}$ foils, it was not enough to fully resolve the discrepancy. The incident beam energy would have to be as low as $97.1 \mathrm{MeV}$ during the time of the experiment to fully resolve the activity discrepancy in all foils, provided the sole source of the discrepancy was attributed to the value of primary beam energy. Without a concurrent timeof-flight energy measurement, this $97.1 \mathrm{MeV}$ incident energy can only be speculative.

\subsection{Future Work}

The calculated fluence as a function of foil number, presented in TABLE 1, conforms to the 7$10 \%$ attenuation expected for the foil stack. There is a fairly small margin of uncertainty for the fluence in the front of the stack, stemming from gamma measurement uncertainty (on the order of $1 \%$ for the first foil) and uncertainty in the cross section (1.2\% for the first foil). Any subsequent uncertainty in the fluence resulting from code predictions cannot be directly quantified without a more extensive experimental effort.

Future research will include further validation of the fluence and its attenuation. The experimental setup at the IPF precludes the use of a direct measurement system, such as a faraday cup. Additional, complementary methods such as isotope ratios [12] may be used to indirectly measure the fluence.

\section{Conclusion}

The use of computational simulations helped us to determine the degraded $100 \mathrm{MeV}$ proton beam's energy more accurately in the energy range between 100 and $50 \mathrm{MeV}$. As the beam degrades below $50 \mathrm{MeV}$, computational simulations diverged from experimentally presumed energies by over-predicting the energy. This observation has been noted in past studies $[15,16]$ that compare measurements using the stacked foil technique with stopping power based calculations.

The various computational models used in this work were able to predict, with good agreement, the energy as a function of depth for complex foil stack geometries. Their predictions 
diverge as the beam energy distribution broadens and statistical uncertainties propagate. A careful inspection of the codes revealed that these discrepancies likely originate from minute differences between the cross sections and stopping power tables that MCNP6 and TRIM/A\&Z use respectively. Energy predictions by TRIM/A\&Z at each foil location that slightly lower than MCNP6 predictions. In all cases, the codes under-predict the energy loss. It should therefore also be noted that a progressive downward shift in the predicted energy values with increasing depth in the stack would also resolve the experiment-computational discrepancy.

\section{Acknowledgements}

We gratefully acknowledge funding for this study provided by the United States Department of Energy, Office of Science via funding from the Isotope Development and Production for Research and Applications subprogram in the Office of Nuclear Physics. Many thanks to the Isotope Production Group, C-IIAC, Rod McCrady, and Heath Watkins in the Accelerator group, AOT, for their guidance and technical ingenuity that made many parts of this study possible.

\section{References}

[1] J. T. Goorley, M. R. James, T. E. Booth, F. B. Brown, and J. S. Bull, "Initial MCNP6 Release Overview- MCNP6 version 1.0," Nucl. Technol., vol. 180, no. 3, pp. 298-315, 2012.

[2] J. F. Ziegler, M. D. Ziegler, and J. P. Biersack, "SRIM - The stopping and range of ions in matter (2010)," Nucl. Instruments Methods Phys. Res. Sect. B Beam Interact. with Mater. Atoms, vol. 268, pp. 1818-1823, 2010.

[3] H. H. Andersen and J. F. Ziegler, Hydrogen Stopping Power and Ranges in All Elements. New York: Pergamon Press, 1977.

[4] J. B. Cumming, "Monitor Reactions for High Energy Proton Beams," Annu. Rev. Nucl. Sci., vol. 13, pp. 261-286, 1963.

[5] J. W. Engle, S. G. Mashnik, H. Bach, A. Couture, K. Jackman, R. Gritzo, B. D. Ballard, M. Fassbender, D. M. Smith, L. J. Bitteker, J. L. Ullmann, M. S. Gulley, C. Pillai, K. D. John, E. R. Birnbaum, and F. M. Nortier, "Cross sections from $800 \mathrm{MeV}$ proton irradiation of terbium," Nucl. Phys. A, vol. 893, pp. 87-100, Nov. 2012.

[6] J. W. Engle, S. G. Mashnik, J. W. Weidner, L. E. Wolfsberg, M. E. Fassbender, K. Jackman, A. Couture, L. J. Bitteker, J. L. Ullmann, M. S. Gulley, C. Pillai, K. D. John, E. R. Birnbaum, and F. M. Nortier, "Cross sections from proton irradiation of thorium at $800 \mathrm{MeV}$," Phys. Rev. C, vol. 88, no. 014604, Jul. 2013.

[7] J. W. Engle, J. W. Weidner, B. D. Ballard, M. E. Fassbender, L. A. Hudston, K. R. Jackman, D. E. Dry, L. E. Wolfsberg, L. J. Bitteker, J. L. Ullmann, M. S. Gulley, C. Pillai, G. Goff, E. R. Birnbaum, K. D. John, S. G. Mashnik, and F. M. Nortier, "Ac, La and Ce radioimpurities in 225Ac produced in 40-200 MeV proton irradiations of thorium.," Radiochim. Acta, vol. 102, no. 7, Jan. 2014.

[8] J. W. Engle, H. Bach, A. Couture, R. Gritzo, D. M. Smith, L. J. Bitteker, J. L. Ullmann, M. Gulley, C. Pillai, E. R. Birnbaum, K. D. John, and F. M. Nortier, "Cross sections for proton induced 
reactions on terbium at $200 \mathrm{MeV}, "$ in AIP Conference Proceedings of the 14th Workshop on Targetry and Target Chemistry, 2012, vol. 152, pp. 152-156.

[9] J. W. Weidner, S. G. Mashnik, K. D. John, F. Hemez, B. Ballard, H. Bach, E. R. Birnbaum, L. J. Bitteker, a. Couture, D. Dry, M. E. Fassbender, M. S. Gulley, K. R. Jackman, J. L. Ullmann, L. E. Wolfsberg, and F. M. Nortier, "Proton-induced cross sections relevant to production of 225Ac and 223Ra in natural thorium targets below 200MeV," Appl. Radiat. Isot., vol. 70, no. 11, pp. 2602-2607, Nov. 2012.

[10] G. F. Steyn, S. J. Mills, F. M. Nortier, B. R. S. Simpson, and B. R. Meyer, "Production Reactions of Fe-52 via Proton-induced on Manganese and Nickel," Appl. Radiat. Isot., vol. 41, no. 3, pp. 315-325, 1990.

[11] F. Tarkanyi, S. Takacs, K. Gul, and A. Hermanne, "Beam Monitor Reactions," in IAEA-TECDOC1211: Charged particle cross-section database for medical radioisotope production: diagnostic radioisotopes and monitor reactions, no. May, Vienna, 2001, p. 49.

[12] K. Gagnon, M. Jensen, H. Thisgaard, J. Publicover, S. Lapi, S. a McQuarrie, and T. J. Ruth, "A new and simple calibration-independent method for measuring the beam energy of a cyclotron.," Appl. Radiat. Isot., vol. 69, no. 1, pp. 247-53, Jan. 2011.

[13] F. Janni, "Proton Range-Energy Tables, 1 keV-10 GeV, Energy Loss, Range, Path Length, Timeof-Flight, Straggling, Multiple Scattering, and Nuclear Interaction Probability," At. Data Nucl. Data Tables, vol. 27, p. 147, 1982.

[14] S. G. Mashnik, K. K. Gudima, R. E. Prael, A. J. Sierk, M. I. Baznat, and N. V Mokhov, "CEM03.03 and LAQGSM03.03 Event Generators for the MCNP6, MCNPX, and MARS15 Transport Codes," in Joint ICTP-IAEA Advanced Workshop on Model Codes for Spallation Reactions, 2008, no. April, p. LANL Report LA-UR-08-2931.

[15] J. H. Kim, H. Park, S. Kim, J. S. Lee, and K. S. Chun, "Proton Beam Energy Measurement with the Stacked Cu Foil Technique for Medical Radioisotope Production," J. Korean Phys. Soc., vol. 48, no. 4, pp. 755-758, 2006.

[16] M. U. Khandaker, G. Kim, K. Kim, H. B. A. Kassim, and B. Nikouravan, "Investigations of proton beam energy of the MC-50 cyclotron at KIRAMS," Int. J. Phys. Sci., vol. 6, no. 13, pp. 31683174, 2011.

LA-UR-14-24373 